\author{
Sandro PiAZZINI* - Leonardo FAVILLI*
}

\title{
Recenti acquisizioni sulla fauna a Lepidotteri Ropaloceri del Parco Nazionale delle Foreste Casentinesi, Monte Falterona e Campigna (Emilia-Romagna e Toscana) ${ }^{1}$
}

\begin{abstract}
Riassunto: Negli anni 2012-2014 è stato raccolto un cospicuo numero di dati faunistici sui Ropaloceri del Parco Nazionale delle Foreste Casentinesi, Monte Falterona e Campigna (Emilia-Romagna e Toscana). Questi dati contribuiscono a definire meglio la distribuzione di 17 specie rare o poco comuni e, nel caso di Melitaea aurelia Nickerl, 1850 e di Apatura ilia ([Denis \& Schiffermüller], 1775), rappresentano la prima segnalazione per il territorio del Parco.
\end{abstract}

\begin{abstract}
Recent acquisitions on the rhopaloceran fauna of the Parco Nazionale delle Foreste Casentinesi, Monte Falterona and Campigna (Emilia-Romagna and Tuscany).

In years 2012-2014 we have made many records of Rhopalocera of Parco Nazionale delle Foreste Casentinesi, Monte Falterona e Campigna (Emilia-Romagna and Tuscany, Italy). These records contribute to the distribution of 14 rare or uncommon species and in case of Melitaea aurelia Nickerl, 1850 and Apatura ilia ([Denis \& Schiffermüller], 1775), are the first reports from the National Park.
\end{abstract}

Key words: Rhopalocera, Parco Nazionale delle Foreste Casentinesi, Monte Falterona e Campigna (Emilia-Romagna and Tuscany, Italy), New records.

\section{INTRODUZIONE}

Nel 2012 il Parco Nazionale delle Foreste Casentinesi, Monte Falterona e Campigna ha attivato, in collaborazione con il Dipartimento di Scienze Fisiche della Terra e dell'Ambiente dell'Università di Siena, un progetto biennale di monitoraggio della fauna minore (Invertebrati, Anfibi, Rettili, Chirotteri e Micromammiferi) vivente nel versante adriatico del suo territorio, con l'intento di dotarsi degli strumenti per gestire le entità e le popolazioni di maggior interesse conservazionistico.

$\mathrm{Nel}$ corso di questa ricerca sono stati raccolti numerosi dati su specie fino ad oggi segnalate per un numero estremamente limitato di località o nuove per il Parco, che aggiornano le conoscenze sulla fauna di questo comprensorio. In questa nota vengono presentati i dati relativi ai Lepidotteri Ropaloceri.

\section{MATERIALI E METODI}

Sono state visitate 91 località (Fig. 1) che coprono tutte le tipologie ambientali presenti nell'area di studio. I campionamenti sono stati effettuati nel periodo maggio-settembre degli anni 2012-2014, privi- legiando per le osservazioni le ore centrali della giornata (tra le 10:00 e le 16:00), durante le quali i lepidotteri risultano più attivi e, quindi, più facili da rilevare. Gli esemplari sono stati catturati con retini entomologici, determinati e immediatamente rilasciati. $\mathrm{Nel}$ caso di specie il cui riconoscimento è possibile solo osservando gli apparati genitali, è stato utilizzato il metodo proposto da Lafranchis (2004), che permette di esaminare gli stessi direttamente sul campo, ricorrendo a una lente di ingrandimento.

Tutti i materiali campionati sono stati determinati facendo riferimento a Tolman \& Lewington (2009), Villa et al. (2009) e van Oorschot \& Coutsis (2014).

Per ciascuna specie vengono riportati: i "Reperti", elencati indicando località, particella del reticolato UTM $1 \times 1 \mathrm{~km}$ (toponimi e riferimenti UTM sono stati tratti dalla Carta Topografica d'Italia dell'IGMI scala 1:25.000 Serie M 891), altitudine, comune e sigla della provincia (tra parentesi), rilevatore/i (DA: Davide Alberti; GC: Giovanni Cappelli; GT: Giuseppe Taras; LA: Leo Agostini; LAz: Lorenzo Azimonti; LR: Luca Ricigliano; NA: Nevio Agostini; SB: Stefano Baroni; SG: Sarah Gonnelli; SP: Sandro Piaz-

\footnotetext{
*Sandro Piazzini, Leonardo Favilli, Dipartimento di Scienze Fisiche della Terra e dell'Ambiente, Università di Siena, Via Mattioli 4, 53100 Siena, Italia. E-mail: piazzini5@unisi.it; leonardo.favilli@unisi.it

${ }^{1}$ Ricerca effettuata con contributo del Parco Nazionale delle Foreste Casentinesi, Monte Falterona e Campigna (Determinazioni Dirigenziali $\mathrm{n}^{\circ} 569$ del 30.12.2011 e $\mathrm{n}^{\circ} 523$ del 27.12.2012).
} 


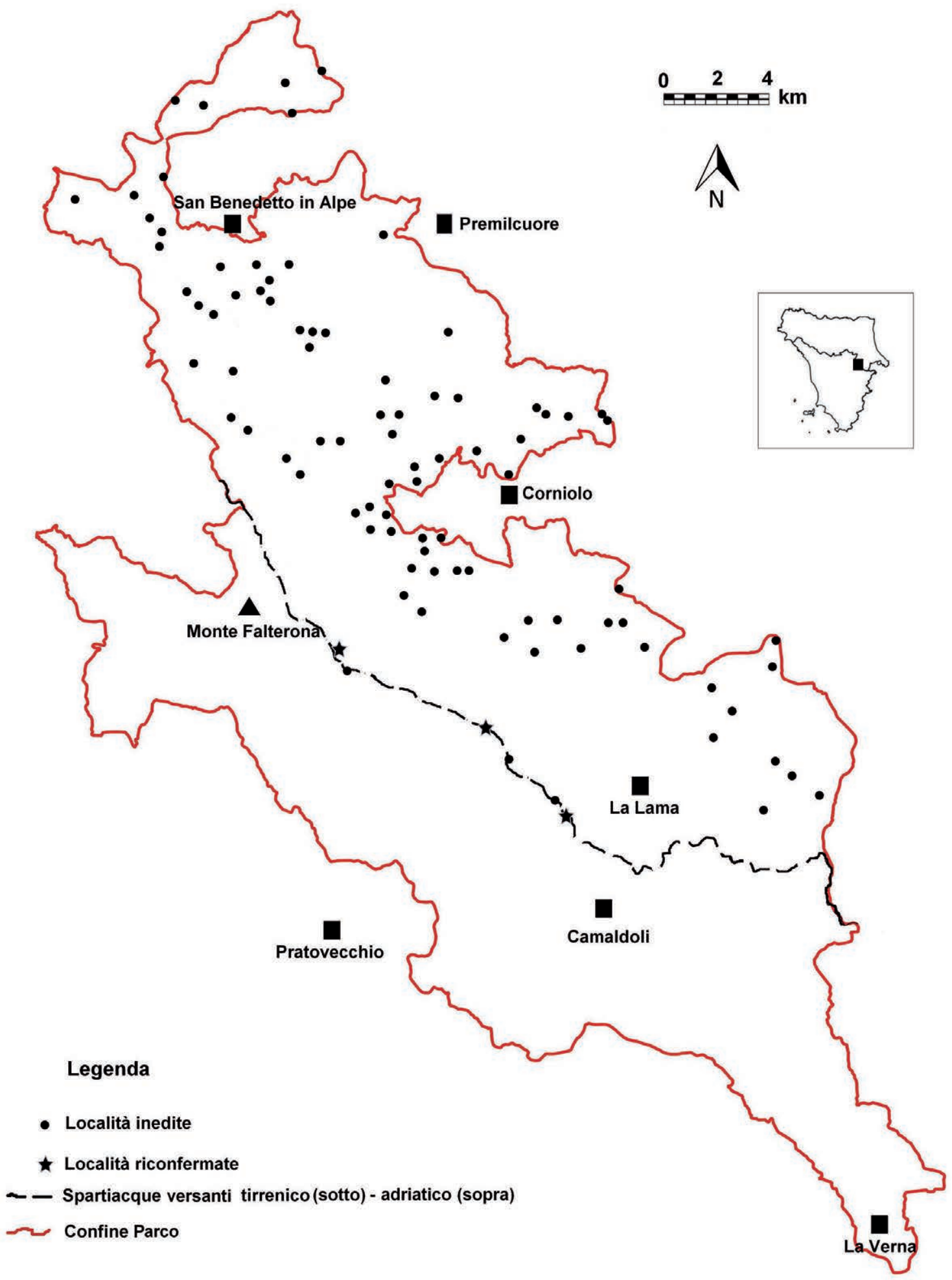

Fig. 1 - Mappa dell'area di studio con indicate le località di campionamento. 
zini), data; le "Osservazioni", relative alla presenza nel Parco prima di questa ricerca e al motivo di interesse della segnalazione.

Tassonomia e nomenclatura scientifica seguono Balletto et al. (2014).

\section{ELENCO DELLE SPECIE}

Pyrgus armoricanus (Oberthür, 1910) (Hesperiidae) RePERTI. Casa Monte di Gralli di sotto, 32TQP1468, 882 m (San Godenzo, FI), SP leg. 21.VII.12; Monte Bucine, $250 \mathrm{~m}$ a SW, 32TQP1671, $1090 \mathrm{~m}$ (Portico e San Benedetto, FC), GC, SP \& GT leg. 18.IX.12; Casa Il Bucine, 32TQP1771, $956 \mathrm{~m}$ (Premilcuore, FC), SP leg. 23.VIII.12; Pian di Rocchi, 32TQP2168, 828 m (Premilcuore, FC), SP leg. 9.IX.12; Monte dell'Avorgnolo, 32TQP2266, 1165 m (Premilcuore-Santa Sofia, FC), SP leg. 1.IX.13; La Bertesca, $50 \mathrm{~m}$ a S, 32TQP3256, 930 m (Bagno di Romagna, FC), SP leg. 22.VIII.12.

OSSERVAZIONI. P. armoricanus era noto in due località: San Benedetto in Alpe e dintorni di Pratovecchio (Zangheri, 1966; Dapporto et al., 2004, 2005). I nostri reperti si riferiscono a sei nuove località del versante adriatico del Parco.

Carcharodus alceae (Esper, [1780]) (Hesperiidae) RePERTI. Casa Frassine, $50 \mathrm{~m}$ a S, 32TQP1766, $838 \mathrm{~m}$ (San Godenzo, FI), SP leg. 19.VII.12; Valbiancana di sotto, $300 \mathrm{~m}$ a NE, 32TQP1866, $900 \mathrm{~m}$ (Premilcuore, FC), LA, MB \& SP leg. 2.VIII.12; Fosso Pian di Soia, ponte S.S. 67 Tosco-Romagnola, 32TQP1471, $622 \mathrm{~m}$ (San Godenzo, FI), SP leg. 23.VI.12; Fosso di Amedani, confluenza Fosso di Mugnai, 32TQP1870, 677 $\mathrm{m}$ (Premilcuore, FC), LA, NA, MB \& SP leg. 10.VII.12; Vecchio vivaio di Corniolo, 32TQP2366, $605 \mathrm{~m}$ (Santa Sofia, FC), SP leg. 7.VIII.13; Mandriolo di sotto, $120 \mathrm{~m}$ a SE, 32TQP2568, $875 \mathrm{~m}$ (Santa Sofia, FC), SP leg. 7.VIII.13.

OsSERVAZIONI. C. alceae era noto in cinque località, le prime due delle quali esterne ai confini del Parco: Corniolo, Isola loc. Spugna, sopra Stia, Camaldoli e Passo della Calla (Zangheri, 1966; Dapporto et al., 2004, 2005). I nostri reperti si riferiscono a sei nuove località del versante adriatico del Parco.

Carcharodus floccifer (Zeller, 1847) (Hesperiidae) RePERTI. Prato al Soglio, 32TQP1656, 1342 m (PoppiBagno di Romagna, AR-FC), SP leg. 21.VII.13; Casa Frassine, $50 \mathrm{~m}$ a S, 32TQP1766, $838 \mathrm{~m}$ (San Godenzo, FI), SP leg. 19.VII.12; Gli Acquastrini, 32TQP1472,
$610 \mathrm{~m}$ (Portico e San Benedetto, FC), SP leg. 26.VII.12; La Lama, chiesa, 32TQP2857, $715 \mathrm{~m}$ (Bagno di Romagna, FC), LA, MB, SP \& LR leg. 15.VII.13; Mandriacce, $150 \mathrm{~m}$ a N, 32TQP2163, 875 m (Santa Sofia, FC), SP leg. 7.VIII.13.

OsSERVAZIONI. C. floccifer è considerato "Near Threatened" in Europa (van Swaay et al., 2010) e a "Minor Preoccupazione" in Italia (Balletto et al., 2016). Fino a oggi era conosciuto in quattro località: Pratovecchio, Passo del Muraglione, Campigna e Passo della Calla (Zangheri, 1966; Fiumi \& Camporesi, 1988; Dapporto et al., 2004, 2005). I nostri reperti si riferiscono a cinque nuove località del versante adriatico del Parco.

Thymelicus lineola (Ochsenheimer, [1808]) (Hesperiidae)

REPERTI. Valbiancana di sotto, $300 \mathrm{~m}$ a NE 32TQP1866, $900 \mathrm{~m}$ (Premilcuore, FC), SP leg. 28.VI.12; Casa Torni, $150 \mathrm{~m}$ a SSE, 32TQP1964, 980 m (Santa Sofia, FC), SG \& SP leg. 6.VII.13; Balze di Cornacchiaia, 32TQP1074, $1050 \mathrm{~m}$ (San Godenzo, FI), SP leg. 5.VII.12; Casa Pian Baruzzoli, $250 \mathrm{~m}$ a E, 32TQP1374, $840 \mathrm{~m}$ (Portico e San Benedetto, FC), SP leg. 5.VII.12; Pian d'Astura, 32TQP1672, 810 m (Portico e San Benedetto, FC), SP leg. 21.VI.12; Monte Collina, 32TQP1777, 940 $\mathrm{m}$ (Tredozio, FC), SP leg. 9.VII.12; Leprata, $120 \mathrm{~m}$ a SW, 32TQP1878, $600 \mathrm{~m}$ (Tredozio, FC), SP leg. 9.VII.12; La Lama, chiesa, 32TQP2857, $715 \mathrm{~m}$ (Bagno di Romagna, FC), LA, MB, SP \& LR leg. 15.VII.13; Monte Cavallo, $300 \mathrm{~m}$ a W, 32TQP2066, $1050 \mathrm{~m}$ (Santa Sofia, FC), SP leg. 14.VII.13; Lavacchio di sopra, 32TQP2166, $1060 \mathrm{~m}$ (Santa Sofia, FC), SP leg. 14.VII.13; Cimitero di San Paolo in Alpe, 32TQP2462, $971 \mathrm{~m}$ (Santa Sofia, FC), SP leg. 13.VII.13; Mandriolo di sopra, $120 \mathrm{~m}$ a E, 32TQP2468, 990 m (Santa Sofia, FC), LA, NA, MB, SP \& LR leg. 16.VII.13; Poggio Fabbreria, $100 \mathrm{~m}$ a N, 32TQP2568, 950 m (Santa Sofia, FC), LA, NA, MB, SP \& L. Ricigliano leg. 16.VII.13; Ridracoli, 32TQP2763, 420 m (Bagno di Romagna, FC), SP leg. 22.VI.13; Casanova dell'Alpe, 32TQP3060, 975 m (Bagno di Romagna, FC), SP leg. 29.VI.12.

OsSERVAZIONI. T. lineola era noto in quattro località, la prima delle quali esterna ai confini del Parco: Isola loc. Spugna, San Benedetto in Alpe, Passo dei Mandrioli e Casanova dell'Alpe (Zangheri, 1966; Fiumi \& Camporesi, 1988; Dapporto et al., 2004, 2005). I nostri reperti si riferiscono a quindici nuove località del versante adriatico del Parco. 
Parnassius mnemosyne (Linné, 1758) (Papilionidae) RePERTI. Prato al Soglio, 32TQP1656, 1342 m (PoppiBagno di Romagna, AR-FC), SP leg. 30.VI.13; Prati della Burraia, 32TQP1960, 1470 m (Santa Sofia, FC), SP leg. 3.VII.13; Monte Gabrendo, 32TQP1960, 1525 m (Stia-Santa Sofia, AR-FC), SP leg. 3.VII.13; Passo del Porcareccio, $350 \mathrm{~m}$ a SE, 32TQP2457, $1385 \mathrm{~m}$ (Bagno di Romagna, FC), SP leg. 30.VI.13; Poggio Scali, 32TQP2458, 1520 m (Pratovecchio-Santa SofiaBagno di Romagna, AR-FC), SP, leg. 30.VI.13; Giogo Secchieta, 32TQP2556, 1380 m (Poppi-PratovecchioBagno di Romagna, AR-FC), SP leg. 30.VI.13.

OSSERVAZIONI. $P$. mnemosyne è ritenuto "Near Threatened" in Europa (van Swaay et al., 2010) e a "Minor Preoccupazione" in Italia (Balletto et al., 2016). Fino a oggi era conosciuto in dieci località: Campigna, Fangacci, Monte Falterona, Prato Bertone, Prato alla Penna, Prato al Soglio, Poggio Scali, La Burraia, Monte Falco e Passo della Calla (Verity, 1947-1950; Zangheri, 1966; Zangheri, 1985; Fiumi \& Camporesi, 1988; Dapporto et al., 2004, 2005). I nostri reperti documentano la sua presenza in due nuove località, Giogo Secchieta e Fonte del Porcareccio, e la riconfermano a Poggio Scali, Prato al Soglio e La Burraia, ma non a Campigna, Prato Bertone, Prato alla Penna, Monte Falco e al Passo della Calla. È possibile che sia scomparsa da queste cinque località per la notevole riduzione delle praterie dovuta alla cessazione del pascolo del bestiame e alla ricolonizzazione da parte delle faggete.

Iphiclides podalirius (Linnaeus, 1758) (Papilionidae) RePERTI. Casa Monte di Gralli di sotto, 32TQP1468, $882 \mathrm{~m}$ (San Godenzo, FI), SP leg. 21.VII.12; Forcone, 32TQP1567, 855 m (San Godenzo, FI), SP leg. 4.VII.12; Casa Frassine, $50 \mathrm{~m}$ a S, 32TQP1766, $838 \mathrm{~m}$ (San Godenzo, FI), SP leg. 19.VII.12; Valbiancana di sotto, $300 \mathrm{~m}$ a NE 32TQP1866, $900 \mathrm{~m}$ (Premilcuore, FC), LA, MB \& SP leg. 2.VIII.12; Casa La Fossa, 32TQP1964, 850 m (Santa Sofia, FC), SG \& SP leg. 6.VII.13; Casa Val di Sparviera, 32TQP1967, $930 \mathrm{~m}$ (Premilcuore, FC), SP leg. 19.VII.12; Trafossi, 32TQP1373, 724 m (San Benedetto e Portico, FC), SP leg. 3.VIII.12; Casa Brensica, 32TQP1374, 650 m (San Benedetto e Portico, FC), SP leg. 26.VII.12; Campo del Fango, $400 \mathrm{~m}$ a N, 32TQP1377, $840 \mathrm{~m}$ (San Benedetto e Portico, FC), SP leg. 26.VII.12; Gli Acquastrini, 32TQP1472, 610 m (San Benedetto e Portico, FC), SP leg. 26.VII.12; Migliorina, 32TQP1570, $880 \mathrm{~m}$ (San Godenzo, FI), SP leg. 3.VIII.12; Pian d'Astura, $750 \mathrm{~m}$ a SSE, 32TQP1671, 985 m (San Benedetto e Portico,
FC), SP leg. 21.VII.12; Pian d'Astura, 32TQP1672, $810 \mathrm{~m}$ (San Benedetto e Portico, FC), SG \& SP leg. 21.VII.12; Casa Il Bucine, 32TQP1771, $956 \mathrm{~m}$ (Premilcuore, FC), LA, NA, MB \& SP leg. 10.7.12; SP, 23.VIII.12; Cà Amedani 32TQP1870, 750 m (Premilcuore, FC), LA, NA, MB \& SP leg. 10.VII.12; Casa Vecchia, $650 \mathrm{~m}$ a SE, 32TQP1872, $1030 \mathrm{~m}$ (San Benedetto e Portico, FC), SP leg. 8.VIII.12; Fiume Bidente delle Celle, $400 \mathrm{~m}$ a N di Partinico, 32TQP2064, $660 \mathrm{~m}$ (Santa Sofia, FC), SP leg. 2.VIII.13; Case Montecavallo di sopra, $100 \mathrm{~m}$ a N, 32TQP2065, $1000 \mathrm{~m}$ (Santa Sofia, FC), SP leg. 9.V.13; Torrente Fiumicello, $250 \mathrm{~m}$ a E di Cà di Broglio, 32TQP2068, 645 m (Premilcuore, FC), LA, MB \& SP leg. 2.VIII.12; Mandriacce, $150 \mathrm{~m}$ a N, 32TQP2163, $875 \mathrm{~m}$ (Santa Sofia, FC), LA, MB \& SP leg. 14.6.13; SP leg. 7.VIII.13; Casa Filettino, 32TQP2164, 665 m (Santa Sofia, FC), SP leg. 2.VIII.13; Lavacchio di sopra, 32TQP2166, 1060 m (Santa Sofia, FC), SP leg. 7.VIII.13; Pian di Rocchi, 32TQP2168, $828 \mathrm{~m}$ (Premilcuore, FC), SP leg. 19.VIII.12; Martinaccio, $200 \mathrm{~m}$ a SE, 32TQP2263, 860 m (Santa Sofia, FC), SP leg. 7.VIII.13; Monte dell'Avorgnolo, 32TQP2266, 1165 m (Premilcuore-Santa Sofia, FC), SP leg. 7.VIII.13; Ponte Garella, 32TQP2268, $745 \mathrm{~m}$ (Premilcuore, FC), SP leg. 19.VII.12; Cà Moscoso, 32TQP2363, 636 m (Santa Sofia, FC), SP leg. 4.VIII.13; Vecchio vivaio di Corniolo, 32TQP2366, $605 \mathrm{~m}$ (Santa Sofia, FC), SP leg. 7.VIII.13; Val di Noce, 32TQP2367, 785 m (Santa Sofia, FC), SP leg. 7.VIII.13; Cimitero di San Paolo in Alpe, 32TQP2462, $971 \mathrm{~m}$ (Santa Sofia, FC), SP leg. 13.6.13; GC, SP \& GT leg. 12.VIII.13; Mandriolo di sopra, $120 \mathrm{~m}$ a E, 32TQP2468, $990 \mathrm{~m}$ (Santa Sofia, FC), SP leg. 21.V.13, LA, MB \& SP leg. 14.6.13; Le Pozzacchere $350 \mathrm{~m}$ a NE, 32TQP2561, $930 \mathrm{~m}$ (Santa Sofia, FC), SP leg. 13.VI.13; Mandriolo di sotto, 120 $\mathrm{m}$ a SE, 32TQP2568, $875 \mathrm{~m}$ (Santa Sofia, FC), SP leg. 7.VIII.13; Fiume Bidente di Ridracoli, $400 \mathrm{~m}$ a SW di Le Caselle, 32TQP2862, 450 m (Bagno di Romagna, FC), SP leg. 23.VII.13; Romiceto, 32TQP3058, $935 \mathrm{~m}$ (Bagno di Romagna, FC), SP leg. 6.VIII.13; Pietrapazza, 32TQP3358, 611 m (Bagno di Romagna, FC), SP leg. 18.VII.12.

OSSERVAZIONI. I. podalirius era noto in otto località: Porciano, Pratovecchio, sopra Stia, Serravalle, Moggiona, Case Vellano, Campigna e Poggio Scali (Zangheri, 1966; Fiumi \& Camporesi, 1988; Dapporto et al., 2004, 2005). I nostri reperti si riferiscono a trentasette nuove località del versante adriatico e attestano come la specie, contrariamente a quanto sostenuto da 
Dapporto et al. (2005), sia tutt'altro che poco frequente nel Parco.

\section{Pieris edusa (Fabricius, 1777) (Pieridae)}

RePERTI. Casa Frassine, $50 \mathrm{~m}$ a S, 32TQP1766, $838 \mathrm{~m}$ (San Godenzo, FI), SP leg. 19.VII.12; Casa Pian Baruzzoli, $250 \mathrm{~m}$ a E, 32TQP1374, $840 \mathrm{~m}$ (Portico e San Benedetto, FC), SP leg. 24.V.12, 5.7.12; Monte del Prato Andreaccio, 32TQP1375, 975 m (San GodenzoPortico e San Benedetto, FI-FC), SP leg. 23.VI.12; Casa Rio del Faggio, 32TQP1471, 670 m (San Godenzo, FI), SP leg. 23.V.12; Casa I Piani, 32TQP1870, $700 \mathrm{~m}$ (Premilcuore, FC), LA, NA, MB \& SP leg. 10.VII.12; Ronco del Cianco, $250 \mathrm{~m}$ a ENE, 32TQP2361, $800 \mathrm{~m}$ (Santa Sofia, FC), SP leg. 13.VII.13; La Bertesca, $50 \mathrm{~m}$ a S, 32TQP3256, $930 \mathrm{~m}$ (Bagno di Romagna, FC), SP leg. 29.VI.12.

Osservazioni. $P$. edusa era nota in quattro località, la prima delle quali esterna ai confini del Parco: Corniolo, La Burraia, Case Vellano e dintorni di Stia (Zangheri, 1966; Dapporto et al., 2004, 2005). I nostri reperti si riferiscono a sette nuove località del versante adriatico del Parco.

Pieris mannii (Mayer, 1851) (Pieridae)

Reperti. Pian di Castagno, $300 \mathrm{~m}$ a NNW, 32TQP1667, $980 \mathrm{~m}$ (San Godenzo, FI), SP leg. 4.VII.12; Casa Il Bucine, 32TQP1771, 956 m (Premilcuore, FC), LA, NA, MB \& SP leg. 10.VII.12; Monte Collina, 32TQP1777, $940 \mathrm{~m}$ (Tredozio, FC), SP leg. 9.VII.12; Cerreta, 32TQP1778, $760 \mathrm{~m}$ (Tredozio, FC), SP leg. 9.VII.12; Monte Cavallo, $300 \mathrm{~m}$ a W, 32TQP2066, $1050 \mathrm{~m}$ (Santa Sofia, FC), SP leg. 14.VII.13; Torrente Fiumicello, $250 \mathrm{~m}$ a E di Cà di Broglio, 32TQP2068, 645 m (Premilcuore, FC), LA, MB \& SP leg. 2.VIII.12; Torrente Fiumicello, confluenza Fosso di Montecarelli, 32TQP2069, $595 \mathrm{~m}$ (Premilcuore, FC), SP leg. 28.VI.12; Lavacchio di sopra, 32TQP2166, 1060 m (Santa Sofia, FC), SP leg. 14.VII.13; Monte dell'Avorgnolo, 32TQP2266, 1165 m (Premilcuore-Santa Sofia, FC), SP leg. 14.VII.13; Ponte Garella, 32TQP2268, 745 m (Premilcuore, FC), SP leg. 19.VII.12; Ronco del Cianco, $250 \mathrm{~m}$ a ENE, 32TQP2361, $800 \mathrm{~m}$ (Santa Sofia, FC), SP leg. 13.VII.13; Cimitero di San Paolo in Alpe, 32TQP2462, $971 \mathrm{~m}$ (Santa Sofia, FC), SP leg. 13.VII.13; Casa Ronconi, 32TQP2562, 780 m (Santa Sofia, FC), SP leg. 23.VII.13; Mandriolo di sotto, 120 $\mathrm{m}$ a SE, 32TQP2568, 875 m (Santa Sofia, FC), LA, NA, MB, SP \& LR leg. 16.VII.13; La Casetta,
32TQP2861, 650 m (Bagno di Romagna, FC), SP leg. 23.VII.13; Monte Fumarolo, 32TQP2073, $1020 \mathrm{~m}$ (Portico e San Benedetto-Premilcuore, FC), SP leg. 5.VII.12; Monte Moggio, 32TQP2270, 995 m (Premilcuore, FC), SP leg. 19.VIII.12; Fosso del Trogo, $250 \mathrm{~m}$ a ESE di Poggiolo, 32TQP3159, $670 \mathrm{~m}$ (Bagno di Romagna, FC), LA, NA, MB \& SP leg. 25.VII.12; Le Graticce, $400 \mathrm{~m}$ a SW, 32TQP3358, $700 \mathrm{~m}$ (Bagno di Romagna, FC), SP leg. 18.VII.12;

OSSERVAZIONI. P. mannii era conosciuta in cinque località, la prima delle quali esterna ai confini del Parco: Strabatenza, Camaldoli, Prato alla Penna, Passo della Calla e Poggio Scali (Dapporto et al., 2004, 2005). I nostri reperti si riferiscono a diciannove nuove località del versante adriatico del Parco.

Favonius quercus (Linné, 1758) (Lycaenidae) REPERTI. Torrente Fiumicello, $350 \mathrm{~m}$ a E di Casa Val di Sparviera, 32TQP1967, $765 \mathrm{~m}$ (Premilcuore, FC), LA, MB \& SP leg. 2.VIII.12; Gli Acquastrini, 32TQP1472, 610 m (Portico e San Benedetto, FC), SP leg. 26.VII.12 Castagnoli di sopra, $50 \mathrm{~m}$ a NW, 32TQP2162, $780 \mathrm{~m}$ (Santa Sofia, FC), GC, SP \& GT leg. 12.VIII.13; Fiume Bidente delle Celle, confluenza Fosso delle Mandriacce, 32TQP2164, 612 m (Santa Sofia, FC), SP leg. 2.VIII.13; Fosso di Cavina, $125 \mathrm{~m}$ a NE di La Cavina, 32TQP2168, 645 m (Premilcuore, FC), SP leg. 19.VII.12; Ponte Garella, 32TQP2268, 745 m (Premilcuore, FC), SP leg. 19.VII.12; Fiume Bidente di Campigna, $250 \mathrm{~m}$ a W di Cà Moscoso, 32TQP2363, $600 \mathrm{~m}$ (Santa Sofia, FC), SP leg. 4.VIII.13; Vecchio vivaio di Corniolo, 32TQP2366, 605 m (Santa Sofia, FC), SP leg. 7.VIII.13; Val di Noce, 32TQP2367, 785 m (Santa Sofia, FC), SP leg.7.VIII.13; Orto botanico di Valbonella, 32TQP2467, $715 \mathrm{~m}$ (Santa Sofia, FC), SP leg. 7.VIII.13 Molinuzzo, 32TQP2661, $600 \mathrm{~m}$ (Santa Sofia, FC), SP leg. 24.VII.13; Rio Bacine, $500 \mathrm{~m}$ a SW di Galvane, 32TQP2762, 475 m (Bagno di Romagna, FC), SP leg. 23.VII.13; Fosso di Riborsìa, confluenza affluente di sx proveniente da Poggio Montironi, 32TQP2768, 533 m (Santa Sofia, FC), LA, NA, MB, SP \& LR leg. 16.VII.13 Fiume Bidente di Ridracoli, $400 \mathrm{~m}$ a SW di Le Caselle, 32TQP2862, $450 \mathrm{~m}$ (Bagno di Romagna, FC), SP leg. 23.VII.13; Fosso di Rio d'Olmo, confluenza Fosso della Capra, 32TQP3357, 765 m (Bagno di Romagna, FC), SP leg. 18.VII.12; Fiume Bidente di Pietrapazza, confluenza Fosso del Trogo, 32TQP3260, $495 \mathrm{~m}$ (Bagno di Romagna, FC), LA, MB, SG \& SP leg. 7.VIII.12; 
OSSERVAZIONI. F. quercus era conosciuto in sei località, le prime tre delle quali esterne ai confini del Parco: Isola loc. Spugna, Ridracoli, Corniolo, dintorni di Stia, Pratovecchio e Case Vellano (Dapporto et al., 2004, 2005). I nostri reperti si riferiscono a sedici nuove località del versante adriatico del Parco.

Pseudophilotes baton (Bergsträsser, 1779) (Lycaenidae) RePERTI. Valbiancana di sotto, $300 \mathrm{~m}$ a NE, 32TQP1866, $900 \mathrm{~m}$ (Premilcuore, FC), SP leg. 28.VI.12; Monte del Prato Andreaccio, 32TQP1375, 975 m (San Godenzo-San Benedetto e Portico, FIFC), SP leg. 23.VI.12; Colle del Tramazzo, $600 \mathrm{~m} \mathrm{a}$ W, 32TQP1487, 910 m (San Benedetto e Portico, FC), 9.VII.12; Casa Il Bucine, 32TQP1771, 956 m (Premilcuore, FC), LA, NA, MB \& SP leg. 10.VII.12; Casa Foscolo, $300 \mathrm{~m}$ a S 32TQP1964, $910 \mathrm{~m}$ (Santa Sofia, FC), SP leg. 2.VIII.13; Monte Cavallo, $300 \mathrm{~m}$ a W, 32TQP2066, $1050 \mathrm{~m}$ (Santa Sofia, FC), SP leg. 1.IX.13; Lavacchio di mezzo, $250 \mathrm{~m}$ a NE, 32TQP2166, 975 m (Santa Sofia, FC), SP leg. 9.V.13; Lavacchio di sopra, 32TQP2166, $1060 \mathrm{~m}$ (Santa Sofia, FC), SP leg. 1.IX.13; Mandriolo di sopra, $120 \mathrm{~m} \mathrm{a} \mathrm{E,}$ 32TQP2468, $990 \mathrm{~m}$ (Santa Sofia, FC), SP leg. 16.VII.13; Le Pozzacchere $350 \mathrm{~m}$ a NE, 32TQP2561, $930 \mathrm{~m}$ (Santa Sofia, FC), SP leg. 13.VII.13; Monte Fumarolo, 32TQP2073, 1020 m (San Benedetto e Portico-Premilcuore, FC), SP leg. 5.VII.12.

Osservazioni. $P$. baton era noto in otto località, tutte al di sotto di $800 \mathrm{~m}$ di quota: Isola loc. Spugna, Ridracoli, San Benedetto in Alpe, Case Vellano, Pratovecchio, dintorni di Stia, Porcino e Moggiona (Zangheri, 1966; Fiumi \& Camporesi, 1988; Dapporto et al., 2004, 2005). I nostri reperti si riferiscono a undici nuove località del versante adriatico, ad altitudini comprese tra 900 e $1060 \mathrm{~m}$, e attestano come la specie, contrariamente a quanto sostenuto da Dapporto et al. (2005), sia ben diffusa fino oltre $1000 \mathrm{~m}$ di quota.

\section{Maculinea arion (Linné, 1758) (Lycaenidae)}

REPERTI. Valbiancana di sotto, $300 \mathrm{~m}$ a NE 32TQP1866, $900 \mathrm{~m}$ (Premilcuore, FC), LA, MB \& SP leg. 2.VIII.12; Casa Foscolo, $300 \mathrm{~m}$ a S 32TQP1964, $910 \mathrm{~m}$ (Santa Sofia, FC), SP leg. 2.VIII.13; Colle del Tramazzo, $600 \mathrm{~m}$ a W, 32TQP1477, $910 \mathrm{~m}$ (Portico e San Benedetto, FC), SP leg. 9.VII.12; Casa Il Bucine, 32TQP1771, 956 m (Premilcuore, FC), LA, NA, MB \& SP leg. 10.VII.12; Cerreta, 32TQP1778, $760 \mathrm{~m}$ (Tredozio, FC), SP leg. 9.VII.12; Cà Amedani, 32TQP1870, 750 m (Premilcuore, FC), LA, NA, MB
\& SP leg. 10.VII.12; Poderone, $150 \mathrm{~m}$ a N, 32TQP2163, 860 m (Santa Sofia, FC), NA \& SP leg. 24.VII.13; Lavacchio di mezzo, $250 \mathrm{~m}$ a NE, 32TQP2166, 975 m (Santa Sofia, FC), SP leg. 14.VII.13; Le Pozzacchere $350 \mathrm{~m}$ a NE, 32TQP2561, 930 m (Santa Sofia, FC), SP leg. 13.VII.13.

OSSERVAZIONI. $M$. arion è considerata "Endangered" in Europa (van Swaay et al., 2010) e a "Minor Preoccupazione" in Italia (Balletto et al., 2016). Fino a oggi era conosciuta in cinque località: San Benedetto in Alpe, Campigna, sopra Stia, Corniolo, Ponte Biforco (Zangheri, 1966; Fiumi \& Camporesi, 1988; Dapporto et al., 2004, 2005). I nostri reperti si riferiscono a nove nuove località del versante adriatico del Parco.

Melitaea aurelia Nickerl, 1850 (Nymphalidae) RePERTI. Casa Il Bucine, 32TQP1771, 956 m (Premilcuore, FC), LA, NA, MB \& SP leg. 10.VII.12, SP leg 15.07.14 ; Mandriolo di sopra, $120 \mathrm{~m}$ a E, 32TQP2468, 990 m (Santa Sofia, FC), LA, NA, MB, SP \& LR leg. 16.VII.13, SP leg. 15.VII.14; Poggio Fabbreria, $100 \mathrm{~m}$ a N, 32TQP2568, $950 \mathrm{~m}$ (Santa Sofia, FC), LA, NA, MB, SP \& LR leg. 16.VII.13, SP leg. 15.VII.14, 1 q rinvenuta morta (coll. S. Piazzini, Casole d'Elsa, Siena).

OsSERVAZIONI. $M$. aurelia è ritenuta "Near Threatened" in Europa (van Swaay et al., 2010) e a "Minor Preoccupazione" in Italia (Balletto et al., 2016). È nuova per il Parco e per la Romagna: al momento è limitata a tre località poste tra 950 e 990 m di quota, caratterizzate dalla presenza di praterie seminaturali ben conservate; queste località sono le più meridionali a oggi note per la specie in Italia (Balletto et al., 2007; Cuvelier \& Casini, 2015).

Melitaea trivia ([Denis \& Schiffermüller], 1775) (Nymphalidae)

RePERTI. Casa Foscolo, $300 \mathrm{~m}$ a S 32TQP1964, $910 \mathrm{~m}$ (Santa Sofia, FC), SP leg. 2.VIII.13; Cà di Massimo, $400 \mathrm{~m}$ a SSW, 32TQP2168, $715 \mathrm{~m}$ (Premilcuore, FC), SB \& SP leg. 19.VII.12; Ponte Garella, 32TQP2268, 745 m (Premilcuore, FC), SB \& SP leg. 19.VII.12.

OSSERVAZIONI. $M$. trivia è considerata "Near Threatened" nei paesi costituenti l'Unione Europea (van Swaay et al., 2010) e a "Minor Preoccupazione" in Italia (Balletto et al., 2016). Fino a oggi era nota in quattro località del versante toscano: Porciano, Camaldoli, sopra Stia e dintorni di Pratovecchio (Dapporto et al., 2004, 2005). I nostri reperti sono i primi per il versante adriatico del Parco. 
Euphydryas provincialis (Boisduval, [1828]) (Nymphalidae)

REPERTI. Ontanelli di sopra, $250 \mathrm{~m}$ a NE, 32TQP1570, 1010 m (San Godenzo, FI), SB \& SP leg. 23.V.12; Podere Montecavallo di sopra, 32TQP2065, $970 \mathrm{~m}$ (Santa Sofia, FC), DA \& SP leg. 7.VI.14.

OSSERVAZIONI. E. provincialis era nota in quattro località del versante toscano, tutte nei dintorni di La Verna: Podere Pratalino $350 \mathrm{~m}$ a ESE, Podere Pratalino $800 \mathrm{~m}$ a S, Podere Pratalino $750 \mathrm{~m}$ a SSE e Monte Faggiolo $250 \mathrm{~m}$ a W (Tellini et al., 2008; Favilli et al., 2012). I nostri reperti sono i primi per il versante adriatico del Parco.

Apatura ilia ([Denis \& Schiffermüller], 1775) (Nymphalidae)

RePerti. Fosso dell'Acquacheta, Ca' del Rospo, 32TQP1274, 635 m (Portico e San Benedetto, FC), LAz leg. 1.VII.13; Bidente delle Celle, $800 \mathrm{~m}$ a S di Montecavallo di sopra, 32TQP2164, 605 m (Santa Sofia, FC), LAz leg. 24.VI.13; Fiume Bidente delle Celle, presso il confine del Parco 32TQP2264, $565 \mathrm{~m}$ (Santa Sofia, FC), SP leg. 14.VII.13; Fosso di Riborsìa, Case Riborsìa, 32TQP2768, 490 m (Santa Sofia, FC), SP leg. 16.VII.13; Bidente di Pietrapazza, Molinaccio, 32TQP3261, 465 m (Bagno di Romagna, FC), LAz leg. 29.VI.13.

OSSERVAZIONI. A. ilia era conosciuta in tre località esterne al Parco, a pochi chilometri dai suoi confini: sopra Stia, Isola loc. Spugna e dintorni di Pratovecchio (Fiumi et al., 2003; Dapporto et al., 2004, 2005). I nostri reperti sono i primi per il territorio del Parco.

Hipparchia semele (Linnaeus, 1758) (Nymphalidae) REPERTI. Pian Casciano di sopra, 32TQP1571, $960 \mathrm{~m}$ (San Benedetto e Portico, FC), GC, SP \& GT leg. 18.IX.12; Monte Bucine, $250 \mathrm{~m}$ a SW, 32TQP1671, 1090 m (San Benedetto e Portico, FC), GC, SP \& GT leg. 18.IX.12; Casa Il Bucine, 32TQP1771, $956 \mathrm{~m}$ (Premilcuore, FC), SP leg. 23.VIII.12; Coloreta, 32TQP1870, $760 \mathrm{~m}$ (Premilcuore, FC), SP leg. 19.VIII.12; Case Montecavallo di sopra, 32TQP2065, 960 m (Santa Sofia, FC), SP leg. 14.VII.13; Monte Cavallo, $250 \mathrm{~m}$ a N, 32TQP2066, $1165 \mathrm{~m}$ (Santa Sofia, FC), SP leg. 14.VII.13; Lavacchio di sopra, 32TQP2166, 1060 m (Santa Sofia, FC), SP leg.
7.VIII.13, 1.IX.13; Pian di Rocchi, 32TQP2168, 828 m (Premilcuore, FC), SP leg. 9.IX.12; Monte dell'Avorgnolo, 32TQP2266, 1165 m (Premilcuore-Santa Sofia, FC), SP leg. 14.VII.13, 7.VIII.13, 1.IX.13; San Paolo in Alpe, 32TQP2462, 1028 m (Santa Sofia, FC), SP leg. 24.VII.13.

OsSERVAZIONI. H. semele fino a oggi era conosciuta in dodici località: Corniolo, La Lama, Passo del Muraglione, Campigna, Poggio Scali, La Burraia, Premilcuore, Casanova dell'Alpe, Case Vellano, Porciano, Moggiona e Passo della Calla (Zangheri, 1966; Fiumi \& Camporesi, 1988; Dapporto et al., 2004, 2005). I nostri reperti si riferiscono a dieci nuove località del versante adriatico e inducono a ritenere che sebbene la specie sia considerata in diminuzione (Dapporto et al., 2005), in realtà sia più diffusa nel Parco di quanto noto.

Hipparchia statilinus (Hufnagel, 1766) (Nymphalidae)

REPERTI. Casa Foscolo, $300 \mathrm{~m}$ a S, 32TQP1964, 910 m (Santa Sofia, FC), SP leg. 22.VIII.13; Pian Casciano di sopra, 32TQP1571, 960 m (Portico e San Benedetto, FC), GC, SP \& GT leg. 18.IX.12; Casa Il Bucine, 32TQP1771, $956 \mathrm{~m}$ (Premilcuore, FC), SP leg. 23.VIII.12; Coloreta, 32TQP1870, $760 \mathrm{~m}$ (Premilcuore, FC), SP leg. 19.VIII.12; Monte Cavallo, $250 \mathrm{~m}$ a N, 32TQP2066, 1165 m (Santa Sofia, FC), SP leg. 1.IX.13; Lavacchio di sopra, 32TQP2166, $1060 \mathrm{~m}$ (Santa Sofia, FC), SP leg. 1.IX.13; Pian di Rocchi, 32TQP2168, $828 \mathrm{~m}$ (Premilcuore, FC), SP leg. 9.IX.12; Monte dell'Avorgnolo, 32TQP2266, $1165 \mathrm{~m}$ (Premilcuore-Santa Sofia, FC), SP leg. 1.IX.13; Mandriolo di sopra, $120 \mathrm{~m} \mathrm{a} \mathrm{E,} \mathrm{32TQP2468,} 990 \mathrm{~m}$ (Santa Sofia, FC), SP leg. 1.IX.13.

OSSERVAZIONI. H. statilinus è ritenuta "Near Threatened" in Europa (van Swaay et al., 2010) e a "Minor Preoccupazione" in Italia (Balletto et al., 2016). Fino ad oggi era conosciuta in sei località del versante toscano: Ponte Biforco, Molino di Corezzo, Pratovecchio, sopra Stia, Porciano e Moggiona (Dapporto et $a l ., 2004,2005)$. I nostri reperti sono i primi per il versante adriatico del Parco.

\section{RINGRAZIAMENTI}

Un sincero ringraziamento al Prof. G. Manganelli per la lettura critica del manoscritto. 


\section{BIBLIOGRAFIA}

Balletto E., Bonelli S., Barbero F., Casacci L.P., Sbordoni V., Dapporto L., Scalercio S., Zilli A., Battistoni A., Teofili C., Rondinini C., 2016 - Lista Rossa delle farfalle italiane. Ropaloceri. Comitato italiano IUCN e Ministero dell'ambiente e della tutela del territorio e del mare, Roma.

Balletto E., Bonelli S., Cassulo L., 2007 - Insecta Lepidoptera Papilionoidea (Rhopalocera). In: Ruffo S., Stoch F. (eds.), Checklist and distribution of the Italian fauna. Memorie del Museo civico di Storia naturale di Verona, 2. serie, Sezione Scienze della vita, 17 (2006): 257-261, with data on CD-ROM.

Balletto E., Cassulo L., Bonelli S., 2014 - Annotated checklist of the italian butterflies and skippers (Papilionoidea, Hesperiioidea). Zootaxa, 3853: 1-114.

Cuvelier S., CAsini P.M., 2015 - Melitaea aurelia (Nickerl, 1850) en Italie septentrionale (Lepidoptera: Nymphalidae). Nouvelle population et confirmation de la détermination par dissection des genitalia. Lépidoptères Revue de Lépidoptrèristes de France, 24 (60): 9-11.

Dapporto L., Fiorini G., Fiumi G., Flamigni C., 2004 - I macrolepidotteri del Parco Nazionale delle Foreste Casentinesi, del Monte Falterona e di Campigna (Lepidoptera). Memorie della Società entomologica italiana, 83 (1): 179-248.

Dapporto L., Fiorini G., Fiumi G., Govi G., 2005 - Farfalle e Falene. Lepidoptera. In: Agostini N.N., Senni L., Benvenuto C. (eds.), Atlante della Biodiversità del Parco Nazionale delle Foreste Casentinesi. Primo contributo alla conoscenza della biodiversità del Parco Nazionale delle Foreste Casentinesi, 1. Ministero dell'Ambiente e della tutela del territorio, Ente Parco delle Foreste Casentinesi: 113-151, Arezzo.

Favilli L., Piazzini S., Tellini Florenzano G., Perroud B., Manganelli G., 2012 - Nuovi dati sulla distribuzione in Toscana di alcuni lepidotteri diurni rari o poco noti (Hesperoidea, Papilionoidea). Atti della Società toscana di Scienze naturali residente in Pisa Memorie Serie B, 118: 1-8.

FIUMI G., CAMPORESI S., 1998 - I macrolepidotteri. Collana La Romagna naturale 1. Amministrazione provinciale di Forlì, Forli.

Fiumi G., Govi G., Romagnoli G., 2003 - Aggiornamento delle attuali conoscenze sui lepidotteri diurni della Romagna. Quaderno di Studi e Notizie di Storia naturale della Romagna, 18: 109-114.

LAFranchis T., 2004 - Butterflies of Europe. Diatheo, Paris.

Tellini Florenzano G., Favilli L., Piazzini S., Manganelli G., 2008 - Segnalazioni faunistiche italiane (N. 472-475). Euphydryas provincialis (Boisduval, 1828) (Lepidoptera Nymphalidae). Bollettino della Società entomologica italiana, 140 (2): 122-123.

Tolman T., Lewington R., 2009 - Collins Butterfly Guide. The Most Complete Guide to the Butterflies of Britain and Europe. HarperCollins, London.

van Swayy C.A.M., Cuttelod A., Collins S., Maes D., Munguira M.L., Sasic M., Settele J., Verovnik R., Verstrael T., Warren M., Wiemers M., Wynhoff I., 2010 - European Red List of Butterflies. Pubblication Office of the European Union, Luxembourg.

van Oorschot H., Coutsis J.G., 2014 - The genus Melitaea Fabricius, 1807. Taxonomy and systematic with special reference to the male genitalia (Lepidoptera, Nymphalidae, Nymphalinae). Tshikolovets Publications, Pardubice.

VERITY R., 1947-1950 - Le farfalle diurne d'Italia. Marzocco, Firenze 3.1: XVI + 318 pp. (1947); 3.2: 23 tavv. (1950).

Villa R., Pellecchia M., Pesce G.B., 2009 - Farfalle d'Italia. Istituto per i Beni artistici culturali e naturali della Regione Emilia Romagna, Bologna.

ZANGHERI P., 1966 - Repertorio sistematico e topografico della flora e fauna vivente e fossile della Romagna. Memorie del Museo civico di Storia naturale di Verona, 1. Tomo II: 485-854 pp.

ZANGHERI S., 1985 - Sguardo sintetico sulla lepidotterofauna della foresta di Campigna. Bollettino del Museo civico di Storia naturale di Verona, 10: 7-19. 